\title{
Light meson decays from photon-induced reactions with CLAS
}

\author{
Michael C. Kunkel ${ }^{1, \star}$ \\ for the CLAS Collaboration \\ ${ }^{1}$ Forschungszentrum Jülich, Jülich Germany
}

\begin{abstract}
Photoproduction experiments with the CEBAF Large Acceptance Spectrometer CLAS at the Thomas Jefferson National Facility produce data sets with competitive statistics of light mesons. With these data sets, measurements of transition form factors for $\eta, \omega$, and $\eta^{\prime}$ mesons via conversion decays can be performed using the invariant mass distribution of the final state dileptons. Tests of fundamental symmetries and information on the light quark mass difference can be performed using a Dalitz plot analysis of the meson decay. An overview of preliminary results, from existing CLAS data, and future prospects within the newly upgraded CLAS12 apparatus are given.
\end{abstract}

\section{Introduction}

Decays of light mesons provide insight into the structure of the meson. The Light Meson Decay (LMD) group, established at the Thomas Jefferson National Facility with worldwide collaboration, investigates physics pertaining to, but not limited to, transition form factors, anomalous decays and the search for CP violation through Dalitz plot analysis. The presentation given was an overview of the LMD program, recent updates on measurements and an outlook on measurements that can be taken with the CLAS12 detector.

\section{Light meson decay program}

The light meson group was established in 2013. The goal of the group is to investigate properties of light meson decays using data obtained with the CLAS detector. Since decays of hadrons are independent of production, all CLAS data can be used, however there are two experiments that were chosen as flagships for the program, the g11 and g12 experiment. Both experiments use a photon beam incident on a liquid hydrogen target which created photo-induced reactions with photon beam energies of $800 \mathrm{MeV}-3.8 \mathrm{GeV}$ for $\mathrm{g} 11$ and $1.1 \mathrm{GeV}-5.5 \mathrm{GeV}$ for g12. See [1] for a complete list of meson decays the LMD group plans to investigate.

^e-mail: m.kunkel@fz-juelich.de 


\subsection{The radiative decay of the $\eta$ and $\eta^{\prime}$ meson}

Radiative decays of $\eta, \eta^{\prime} \rightarrow \pi^{+} \pi^{-} \gamma$ are tools to explore QCD anomalies, i.e. box anomaly, and pion final state interactions. The radiative decay widths of $\eta^{\prime}$ and $\eta^{\prime}$ can be expressed as [2];

$$
\frac{d \Gamma\left(\eta^{(\prime)} \rightarrow \pi^{+} \pi^{-} \gamma\right)}{d s_{\pi \pi}}=A\left|P\left(s_{\pi \pi}\right) F_{V}\left(s_{\pi \pi}\right) \Gamma_{0}\left(s_{\pi \pi}\right)\right|
$$

where $\Gamma_{0}\left(s_{\pi \pi}\right)$ is denoted as

$$
\Gamma_{0}\left(s_{\pi \pi}\right)=\frac{\kappa\left(M_{\eta^{\prime \prime)}}^{2}-s_{\pi \pi}\right)^{3} s_{\pi \pi}\left(1-\frac{4 M_{\pi}^{2}}{s_{\pi \pi}}\right)^{\frac{3}{2}}}{M_{\eta^{\prime \prime}}^{3}}
$$

with $\kappa$ being a numerical constant. $F_{V}\left(s_{\pi \pi}\right)$ is the pion form factor and $P\left(s_{\pi \pi}\right)$ is written as a polynomial in $s_{\pi \pi}$ as

$$
P\left(s_{\pi \pi}\right)=1+\alpha s_{\pi \pi}+O\left(s_{\pi \pi}^{2}\right)
$$

where $\alpha$ is the variable of measurement. Previous results on the radiative decay for the $\eta$ meson from WASA-at-COSY [3] and KLOE [4] would profit from further measurements. Only one measurement exists for the $\eta^{\prime}$ radiative decay [5]. With the CLAS g11 experiment, both the $\eta$ and $\eta^{\prime}$ radiative decay width will be measured. In Fig. 1a the CLAS g11 data is shown for the particle selection of exclusive $\gamma p \rightarrow p \pi^{+} \pi^{-} \gamma$. Selecting events within a $2.5 \sigma$ range of $\eta^{\prime}$ and applying efficiency corrections, the $s_{\pi \pi}$ distribution is shown in Fig. $1 \mathrm{~b}$ along with preliminary fit results using Eq. 3 and theory predictions [6].

$$
s_{\pi \pi}=m^{2}-2 E_{\gamma} m
$$

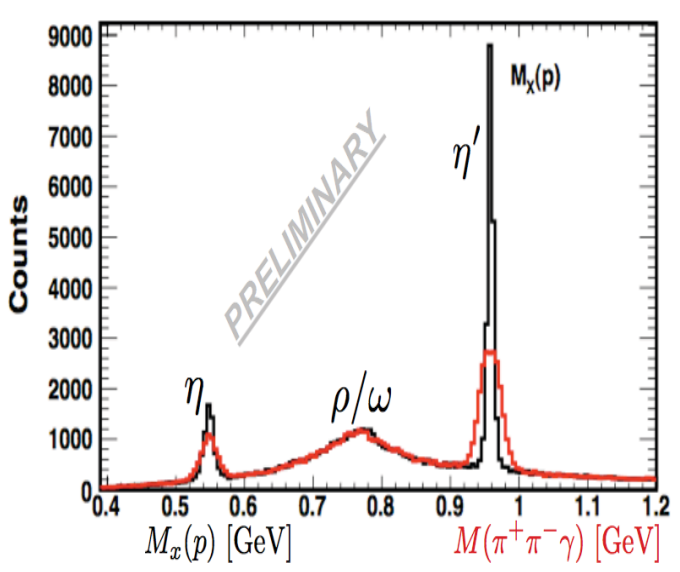

(a)

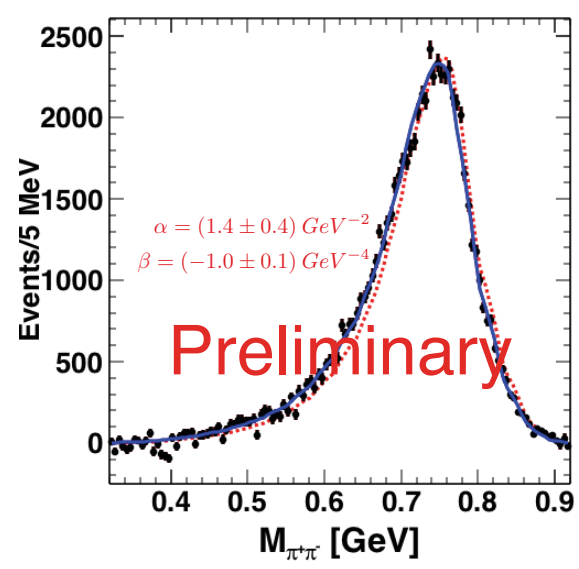

(b)

Figure 1: CLAS yield for $\gamma p \rightarrow p \eta^{(\prime)} \rightarrow p \pi^{+} \pi^{-} \gamma$ from the g11 data set (a).(black) CLAS preliminary $s_{\pi \pi}$ distribution for $\eta^{\prime}$ with (blue solid line) preliminary fit results using Eq.( 3 ) and (red dashed line) theory predictions [6] (b). 


\subsection{Update on the dalitz plot analysis of $\eta^{\prime} \rightarrow \pi^{+} \pi^{-} \eta$}

The Dalitz plot of $\eta^{\prime} \rightarrow \pi^{+} \pi^{-} \eta$ provides kinematic information of the decay, enabling the study of low energy dynamics of QCD and heavier mass pseudoscalar mesons. The $\eta^{\prime} \rightarrow \pi^{+} \pi^{-} \eta$ decay has a low Q-value due to the decay products being relatively heavy, this helps test and limit the effective chiral Lagrangian theory. The measurable of the Dalitz plot $X$ and $Y$ are projected and fitted to

$$
f(X, Y)=A\left(1+a(Y)+b\left(Y^{2}\right)+c(X)+d\left(X^{2}\right)\right.
$$

to extract the parameters $a, b, c, d$. This topic was briefly discussed, as a full update was given by later in the session.

\subsection{The transition form factor of the $\omega$ Meson}

Transition form factors characterize modifications of the point-like photon-meson vertex due to the structure of the meson. The virtual photon can interact with quarks, therefore it can be used as a probe for the internal structure of mesons and its electromagnetic interaction is calculable with the Kroll-Wada formula [7];

$$
\frac{d \Gamma_{M \rightarrow l^{+} l^{-} X}}{d q^{2} d \Gamma_{M \rightarrow X \gamma}}=\left.\frac{\alpha}{3 \pi q^{2}}\left(\left(1+\frac{q^{2}}{m_{M}^{2}-m_{X}^{2}}\right)^{2}-\frac{4 m_{M}^{2} q^{2}}{\left(m_{M}^{2}-m_{X}^{2}\right)^{2}}\right)^{\frac{3}{2}}\left(1-\frac{4 m_{l}^{2}}{q^{2}}\right)^{1 / 2}\left(1+\frac{2 m_{l}^{2}}{q^{2}}\right)\right|_{\text {Q.E.D }} .
$$

where $M$ is the species of meson i.e. $\pi^{0}, \eta, \omega, \eta^{\prime}$, etc., $X$ is the child particle in the decay, $m_{M}$ the mass of the meson, $m_{X}$ the mass of the child particle, $m_{l}$ the mass of the lepton species in the decay, i.e. $e^{ \pm}$or $\mu^{ \pm}$and $q$ being the momentum transfer which is identical to the invariant mass of the dilepton. Deviations of Equation 6 represent the internal structure of the meson for pseudoscalar mesons, while for vector mesons the deviation represents the transition from $M \rightarrow X$. These deviations are the transition form factor $\left|F\left(q^{2}\right)\right|$ and can be determined by comparing Equation 6 to what is measured experimentally.

$$
\left.\frac{d \Gamma_{M \rightarrow l^{+} l^{-} X}}{d q^{2} d \Gamma_{M \rightarrow X \gamma}}\right|_{\text {measured }}=\left.\frac{d \Gamma_{M \rightarrow l^{+} l^{-} X}}{d q^{2} d \Gamma_{M \rightarrow X \gamma}}\right|_{\text {Q.E.D }}\left|F\left(q^{2}\right)\right|^{2} .
$$

Depending on the decay width of the meson of interest, the transition can be modeled as a simple pole,

$$
\left|F\left(q^{2}\right)\right|=\frac{1}{1-\frac{q^{2}}{\Lambda^{2}}}
$$

a complex pole,

$$
\left|F\left(q^{2}\right)\right|^{2}=\frac{\Lambda^{2}\left(\Lambda^{2}+\gamma^{2}\right)}{\left(\Lambda^{2}-q^{2}\right) \Lambda^{2} \gamma^{2}},
$$

or some other function that describes the transition. In both Equation 8 and Equation $9, \Lambda$ is the mass and width of the virtual vector meson, while $\gamma$ is the width of the virtual vector meson. Recent measurements of the transition form factor for $\omega \rightarrow \mu^{+} \mu^{-} \pi^{0}$ have shown unexpected discrepancies with the Vector Dominance Model [8] and recent models of chiral Lagrangian field theory [9], and dispersion theory [10] attempt to predict the contributions of the virtual vector meson, as seen in Fig. 2.

With the CLAS g12 experiment, lepton $\left(e^{ \pm}\right)$identification is done using Cherenkov detectors and electromagnetic calorimetry, providing a $e^{+} e^{-} / \pi^{+} \pi^{-}$rejection of $10^{6}$. Using the $e^{ \pm}$data from CLAS $\mathrm{g} 12$, the $\omega \rightarrow p e^{+} e^{-} \pi^{0}$ transition form factor can be extracted. Preliminary studies have shown that the number of $\omega \rightarrow p e^{+} e^{-} \pi^{0}$ events in CLAS to be $\approx 3 \cdot 10^{3}$. 


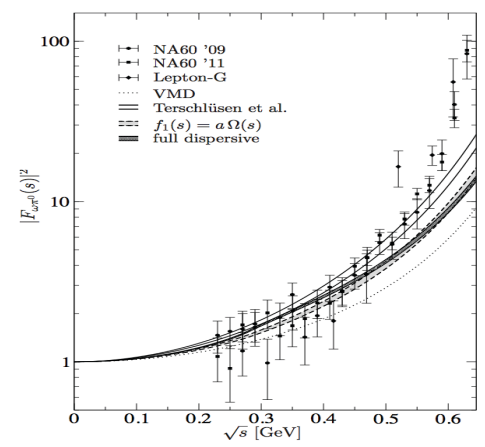

Figure 2: Form factor of the decay $\omega \rightarrow l^{+} l^{-} \gamma$ compared to dimuon data by the NA60 Collaboration [8, $11]$ and LEPS [12]. Shown are VMD (dotted line) Eqs. $(6,9)$ using the mass of the $\rho$ meson as the virtual vector meson, the results of a chiral Lagrangian treatment with explicit vector mesons [9] (white shaded curve with solid borders), a simplified approximation to the full dispersive solution [10] (gray shaded curve with dashed borders), full dispersive solution [10] (black hatched curve with solid borders). [10]

\subsection{Update on the branching ratio measurement of the $\eta^{\prime} \rightarrow e^{+} e^{-} \gamma$ decay}

A recent measurement of BESIII reports the branching ratio $\Gamma\left(\eta^{\prime} \rightarrow e^{+} e^{-} \gamma\right) / \Gamma\left(\eta^{\prime} \rightarrow \gamma \gamma\right)$ to be $2.13 \pm 0.09$ (stat.) \pm 0.07 (sys.) $) 10^{-2}$ from 864 events [13]. Using the $e^{ \pm}$data from CLAS g12, preliminarily 172 events of the $\eta^{\prime} \rightarrow e^{+} e^{-} \gamma$ decay were observed and analyzed, using the Q-factor [14] method to suppress background from neighboring $A \rightarrow e^{+} e^{-} X$ decays, Figure 3. A preliminary branching

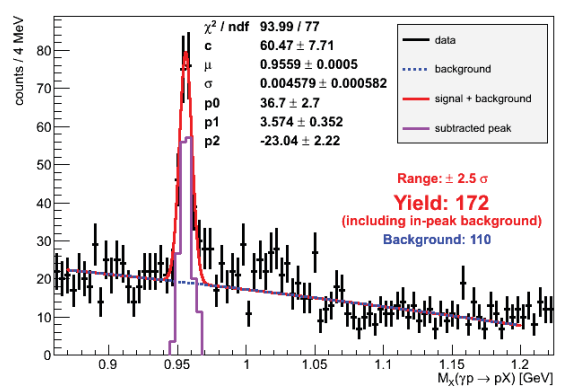

Figure 3: (black)CLAS yield for $\gamma p \rightarrow p \eta^{\prime} \rightarrow p e^{+} e^{-} \gamma$ from g12 data set. (red line) Fit of signal and background using a Voigt signal and 1st order polynomial background function. (magenta line) Background subtracted signal peak, (blue dashed line) represents the background.

ratio $\Gamma\left(\eta^{\prime} \rightarrow e^{+} e^{-} \gamma\right) / \Gamma\left(\eta^{\prime} \rightarrow \gamma \gamma\right)$ was measured to be consistent with the BESIII measurement. However, the statistics of either BESIII or CLAS are not ample enough to determine, statistically, which theoretical model best represents the structure of the $\eta^{\prime}$ meson [15-17]. Table 1 outlines the current measurements and theoretical predictions. 


\begin{tabular}{cc}
\hline Charge Radius $\langle r\rangle$ & Measurement $(\mathrm{M}) /$ Prediction (P) \\
\hline CLAS $\left(\eta^{\prime} \rightarrow e^{+} e^{-} \gamma\right)$ & TBD \\
BESIII $\left(\eta^{\prime} \rightarrow e^{+} e^{-} \gamma\right)$ & (M) $1.60 \pm 0.17($ stat $) \pm 0.08($ sys $) \mathrm{GeV}^{-2}[13]$ \\
CELLO $\left(\eta^{\prime} \rightarrow \mu^{+} \mu^{-} \gamma\right)$ & (M) $1.7 \pm 0.4 \mathrm{GeV}^{-2}[18]$ \\
\hline Dispersion & (P) $1.53_{-0.08}^{0.15} \mathrm{GeV}^{-2}[17]$ \\
ChPT & (P) $1.6 \mathrm{GeV}^{-2}[16]$ \\
VMD & (P) $1.45 \mathrm{GeV}^{-2}[15]$ \\
\hline
\end{tabular}

Table 1: Current Measurements and Theoretical Predictions of the $\eta^{\prime}$ charge radius.

\subsection{Future measurement of the $\eta^{\prime}$ meson transition form factor with CLAS12}

With the newly built CLAS12 detector, $e^{ \pm}$identification can be achieved with a $e^{+} e^{-} / \pi^{+} \pi^{-}$rejection of $10^{6}-10^{11}$ while retaining $e^{+} e^{-} \gamma$ acceptance $\sim 1 \%-0.1 \%$.

Using the CLAS12 FASTMC simulation, a simulation of $10^{6} e p \rightarrow e^{\prime} p \eta^{\prime} \rightarrow p e^{+} e^{-} \gamma\left(e^{\prime}\right)$ was performed. The generation of events included cross-section information obtained from previous CLAS measurements, the $s^{n}$ scaling law on the cross-section and the VMD model for the decay of the $\eta^{\prime}$ meson to achieve a reasonable model of the production of the $\eta^{\prime}$ meson. Estimations of the integrated quasi-real photon rate from an electron beam of luminosity $10^{35} \mathrm{~cm}^{-2} \mathrm{~s}-1$ impinged on a $5 \mathrm{~cm} \ell \mathrm{H}_{2}$ target yielded that the production rate for hadrons to be $80 \mathrm{kHz}$. Therefore, the total rate of $\eta^{\prime}$ can be calculated by defining the following ratio $R(W)$ :

$$
R(W)=\frac{\sigma(W)}{\eta^{\prime} \text { integrated } \sigma(W)}
$$

and scaling the total rate by $R(W)$. This leads to:

$$
\eta^{\prime} \text { total rates } / 80 \text { Days }(W)=80 \mathrm{kHz} \cdot \frac{6.912 \mathrm{e} \cdot 10^{6} \text { seconds }}{80 \text { days }} \cdot \frac{1}{\mathrm{R}(\mathrm{W})}
$$

The total $\eta^{\prime} \rightarrow e^{+} e^{-} \gamma$ rates per 80 days, and as a function $W$, is calculated by multiplying Eq.(11) with the product of the average detection efficiency $\epsilon \approx 5 \%$ as well as the branching fraction $\mathcal{B R}=$ 4.69 $10^{-4}$ [13] for $\eta^{\prime} \rightarrow e^{+} e^{-} \gamma$. The total number $N_{\text {tot }}$ of expected $\eta^{\prime} \rightarrow e^{+} e^{-} \gamma$ events after 80 days of measurement is given by the integral of Eq. (11) over $W$. This leads to the expected yield:

$$
N_{\text {tot }}=\int_{1.9 \mathrm{GeV}}^{2.8 \mathrm{GeV}}\left[N(W)_{\eta^{\prime} \rightarrow e^{+} e^{-} \gamma / 80 \text { Days }}\right] d W=\frac{28,200 \text { events }}{80 \text { Days }}
$$




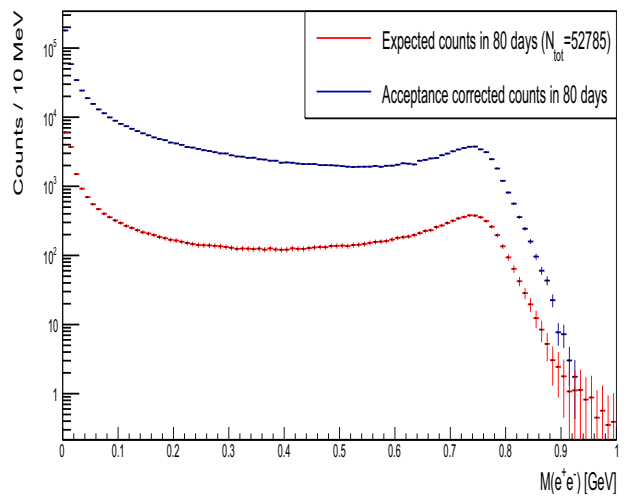

(a)

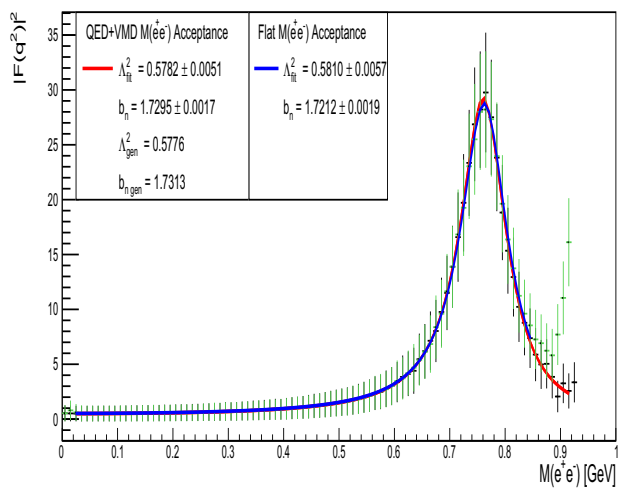

(b)

Figure 4: (Color Online) Expected yield as a function of $M\left(e^{+} e^{-}\right)$(a).(Color Online) $\left|F\left(q^{2}\right)\right|^{2}$ as a function of $M\left(e^{+} e^{-}\right)$using two acceptance models. (black points) QED+VMD $M\left(e^{+} e^{-}\right)$acceptance model. (green points) Flat $M\left(e^{+} e^{-}\right)$acceptance model. The solid lines represent a fit using Eq.(9) to the data points (b).

From Fig. 4a the QED normalized spectrum can be deduced and is shown in Fig. 4b. Both generation models (i.e. flat and QED+VMD) are used to determine the transition form factor. It is shown that there exists a systematic uncertainty depending on the chosen generation model. However, the final calculation on the slope parameter or the TFF shows a negligible impact of this uncertainty. Regardless of the generation model, it is shown in Fig. $4 \mathrm{~b}$ that the accumulated statistics collected by CLAS12 allow for a precision of each parameter $\lesssim 0.5 \%$. Compared to current experimental uncertainties of $10 \%$ and differences in theoretical approaches of $\approx 10 \%$, Table 1 , therefore a measurement by CLAS 12 would not only be in the position to decisively discriminate between the theoretical predictions, but also help to further constrain the hadronic light-by-light amplitude to the muon $g-2$ anomaly [19].

\section{Summary}

The CLAS LMD program covers a variety of meson decay physics. With the data sets collected by the former CLAS detector, transition form factor measurements, investigation of symmetry breaking phenomena and probing QCD anomalies are done with high accuracy. The newly built CLAS12 detector will provide more statistically supported data samples, allowing LMD analyses to probe further into light meson decay physics.

\section{References}

[1] M. Amaryan et al., Photoproduction and Decay of Light Mesons in CLAS

[2] F. Stollenwerk et al., Physics Letters B 707, 184 (2012)

[3] P. Adlarson et al., Physics Letters B 707, 243 (2012)

[4] D. Babusci et al., Physics Letters B 718, 910 (2013) 
[5] A. Abele et al., Physics Letters B 402, 195 (1997)

[6] B. Kubis, J. Plenter, The European Physical Journal C 75, 1 (2015)

[7] N.M. Kroll, W. Wada, Phys. Rev. 98, 1355 (1955)

[8] R. Arnaldi et al., Physics Letters B 677, 260 (2009)

[9] C. Terschlusen, S. Leupold, Physics Letters B 691, 191 (2010)

[10] S.P. Schneider et al., Phys. Rev. D 86, 054013 (2012)

[11] G. Usai, Nuclear Physics A 855, 189 (2011)

[12] R. Dzhelyadin et al., Physics Letters B 102, 296 (1981)

[13] M. Ablikim et al. (BESIII), Phys. Rev. D92, 012001 (2015)

[14] M. Williams et al., JINST 4, P10003 (2009)

[15] A. Bramon, E. Massó, Physics Letters B 104, 311 (1981)

[16] L. Ametller et al., Phys. Rev. D 45, 986 (1992)

[17] C. Hanhart et al., Eur. Phys. J. C 73, 2668 (2013)

[18] R. Dzhelyadin et al., Physics Letters B 88, 379 (1979)

[19] T. Blum et al. (2013), 1311.2198 